\title{
Timing of chemotherapy-induced neutropenia: the prognostic factor in advanced pancreatic cancer patients treated with gemcitabine / gemcitabine-based chemotherapy
}

\author{
Yang Chen ${ }^{1, *}$, Yan Shi ${ }^{1, *}$, Huan Yan ${ }^{1}$, Yan Rong Wang ${ }^{1}$ and Guang Hai Dai ${ }^{1}$ \\ ${ }^{1}$ Medical Oncology Department 2, Chinese PLA General Hospital and Chinese PLA Medical School, Beijing 100853, China \\ * These authors have contributed equally to this work
}

Correspondence to: Guang Hai Dai, email: dgh19661007@126.com

Keywords: advanced pancreatic cancer; timing of chemotherapy-induced neutropenia (CIN); prognosis; chemotherapy Received: June 30, $2016 \quad$ Accepted: March 09, $2017 \quad$ Published: April 09, 2017

Copyright: Chen et al. This is an open-access article distributed under the terms of the Creative Commons Attribution License 3.0 (CC BY 3.0), which permits unrestricted use, distribution, and reproduction in any medium, provided the original author and source are credited.

\section{ABSTRACT}

Chemotherapy-induced neutropenia (CIN) was reported to be a predictor of better survival in several cancers. The objective of our study is to evaluate the relationship between the timing (onset) of CIN and prognosis. Between June 2008 and June 2015, 134 patients with confirmed advanced pancreatic cancer received at least one cycle of gemcitabine / gemcitabine-based chemotherapy as first-line chemotherapy were eligible for assessment. Timing of CIN was categorized into early onset and non-early onset CIN group. The end of cycle 2 was the cutoff to differentiate early onset or non-early onset. The correlation between timing of CIN with survival was analyzed by Kaplan-Meier method and Cox proportional hazards model. Median overall survival (OS) was 8.05 months (95\% CI: 5.97-10.13) for patients with early onset CIN compared with 5.82 months (95\% CI: $5.00-6.63)$ for patients without early-onset neutropenia $(P=0.022)$. Multivariate analysis proved that timing of CIN was an independent prognostic factor, hazard ratios of death was $0.696(95 \%$ CI: 0.466-0.938) for patients with early onset CIN. In conclusion, timing of CIN is an independent predictor of prognosis in patients with advanced pancreatic cancer undergoing gemcitabine / gemcitabine based chemotherapy. Early-onset CIN predicts better survival.

\section{INTRODUCTION}

Pancreatic cancer is responsible for 331,000 deaths per year, and the seventh most common cause of death from cancer in both genders worldwide [1]. Pancreatic cancer is typically diagnosed late, when curative resection is impossible and prognosis is poor, with only $1-2 \%$ of patients surviving at 5 years. Several factors have been proved that could predict survival, such as tumor size, histologic grade, vascular invasion, lymph node metastases and perineural invasion [2]. However, these factors are only available for assessment after surgery. Other novel molecular biomarkers are associated with high costs, time-consuming laboratory experiments. Therefore, there is an urgent need for easily measurable chemotherapy prognostic markers that can be used for stratifying patients' treatment.

Chemotherapy-induced neutropenia (CIN) is a common adverse effect which often results in dose reduction. Several studies have reported the association of CIN with a better clinical outcome in breast cancer, gastric cancer, cervical cancer, unresectable pancreatic cancer and ovarian cancer patients [3-7]. Massimo Di Maio $\mathrm{M}$ et al [8] indicated that the occurrence of CIN is associated with better survival in non small cell lung cancer (NSCLC) patients in a pooled analysis. Although a few studies indicated that CIN was correlated to a better survival, the association between severity of CIN (grade of $\mathrm{CIN}$ ) and prognosis was still controversial. Banerji $\mathrm{U}$ et al [9] proved that patients experienced grade 3-4 neutropenia had a longer time to progress (TTP) and overall survival (OS) than patients experienced grade 1-2 neutropenia in small cell lung cancer (SCLC). Whereas, in Yamanaka T et $a l$ study [10], the gastric cancer patients with severe (grade 3-4) neutropenia did not indicate a better survival.

Recently, Jang SH et al [11] raised a new viewpoint on the correlation between CIN with survival in their study. They found that timing of CIN might be a predictive factor for favorable prognosis in patients with metastatic NSCLC. The predictive or prognostic role of timing of 
CIN has not been established in advanced pancreatic cancer. The objective of this study is to investigate the possible correlation between timing of CIN with prognosis in advanced pancreatic cancer patients.

\section{RESULTS}

\section{Demographics}

Total 134 patients with histologically confirmed advanced pancreatic cancer who received at least one cycle of chemotherapy were eligible for assessment. The median age at diagnosis was 57 years. A total of 30 (22.4\%) patients were locally advanced and 104 (77.6\%) patients had distant metastasis. Among them, 86 (64.2\%) patients experienced early onset CIN and 48 (35.8\%) patients experienced non-early onset CIN. Table 1 shows clinical characteristics by timing of CIN during the firstline chemotherapy of all patients.

\section{Survival analysis}

We subsequently evaluated prognostic significance of timing of CIN using Kaplan-Meier survival analysis, univariate and multivariate Cox regression analysis. The median OS for all patients was 7.06 months, 116 (86.5\%) patients died by end of the follow-up (July $31^{\text {st }}, 2016$ ). Patients with early-onset CIN had a longer survival than those non-early onset CIN (median OS: $8.05 \mathrm{~m}$ vs. $5.82 \mathrm{~m}$, $P=0.022$ ) (Figure 2)

Univariate analysis identified female (female vs. male, $P=0.018$ ), a better tumor pathological differention (poor vs. well-moderate, $P=0.043$ ), patients received gemcitabine and nab-paclitaxel chemotherapy (gemcitabine and nab-paclitaxel vs. gemcitabine monotherapy, $P=0.016$ ), patients with early onset CIN (early onset vs. non-early, $P=0.023$ ) and patients received second-line chemotherapy (yes vs. no, $P=0.019$ ) as better prognostic factors for OS in this study cohort. Age, KPS, location, stage were not significantly associated with clinical outcome (Table 2).

To determine the independent prognostic value of timing of CIN for OS, a multivariate analysis using a Cox proportional hazard model was performed. In the multivariate analysis that included gender, age, KPS, pathology differention, disease extension, first-line chemotherapy regimens, second-line chemotherapy and timing of CIN, we identified timing of CIN (early onset vs. non-early, HR: 0.696 [0.466-0.983],$P=0.027$ ), pathological differention (poor vs. well-moderate, HR: 1.159 [1.078-1.417], $P=0.037$ ) and second-line

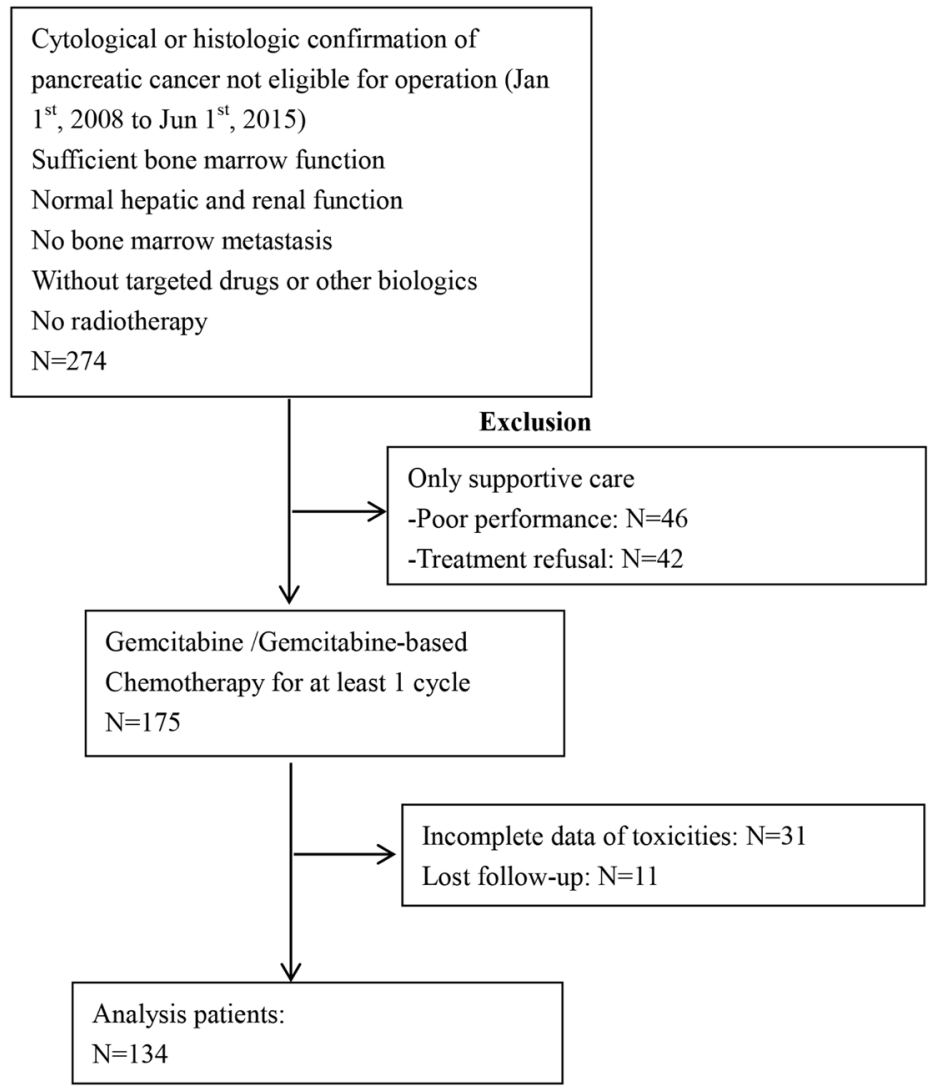

Figure 1: Study flow chart. 
Table 1: Advanced pancreatic cancer patients' characteristics by timing of CIN

\begin{tabular}{|c|c|c|c|c|}
\hline Characteristics & Number of patients $(\%)(n=134)$ & \begin{tabular}{|l} 
Early onset \\
$N=86$
\end{tabular} & $\begin{array}{l}\text { Non-early onset } \\
N=48 \\
\end{array}$ & $P$ value \\
\hline \multicolumn{5}{|l|}{ Age } \\
\hline$\leq 57$ & $73(54.5)$ & 47 & 26 & 0.957 \\
\hline$>57$ & $61(45.5)$ & 39 & 22 & \\
\hline \multicolumn{5}{|l|}{ Gender } \\
\hline Male & $87(65)$ & 51 & 36 & 0.068 \\
\hline Female & $47(35)$ & 35 & 12 & \\
\hline \multicolumn{5}{|l|}{ KPS } \\
\hline 90 & $110(82)$ & 67 & 43 & 0.091 \\
\hline $70-80$ & $24(18)$ & 19 & 5 & \\
\hline \multicolumn{5}{|l|}{ Pathological differention } \\
\hline Well-moderate & $92(69)$ & 53 & 39 & 0.070 \\
\hline poor & $42(31)$ & 33 & 9 & \\
\hline \multicolumn{5}{|l|}{ Disease extension } \\
\hline Locally advanced & $30(22.4)$ & 18 & 12 & 0.588 \\
\hline Distant metastasis & $104(77.6)$ & 68 & 36 & \\
\hline \multicolumn{5}{|l|}{ Location } \\
\hline Head & $44(32.8)$ & 29 & 15 & 0.770 \\
\hline Body-tail & $90(70.2)$ & 57 & 33 & \\
\hline \multicolumn{5}{|c|}{ First-line chemotherapeutic regimens } \\
\hline Gemcitabine monotherapy & $40(29.9)$ & 22 & 18 & 0.084 \\
\hline Gemcitabine and S-1/capecitabine & $19(14.2)$ & 9 & 10 & \\
\hline Gemcitabine and platinum drugs & $6(4.5)$ & 4 & 2 & \\
\hline Gemcitabine and nab-paclitaxel & $69(51.5)$ & 51 & 18 & \\
\hline \multicolumn{5}{|l|}{ Second-line chemotherapy } \\
\hline Yes & $36(27)$ & 24 & 12 & 0.716 \\
\hline No & $98(73)$ & 62 & 36 & \\
\hline
\end{tabular}

Tests used: Wilcoxon test; Pearson test.

chemotherapy (yes vs. no, HR: 0.681 [0.476-0.972] $P=$ 0.035 ) were independent prognostic factors for OS (Table $3)$.

\section{DISCUSSION}

This is the first study, to our knowledge, to prove timing of CIN is a predictor of better prognosis in advanced pancreatic cancer patients undergoing gemcitabine / gemcitabine-based chemotherapy. In our study, median OS was 8.05 months (95\% CI: 5.97-10.13) for patients with early onset CIN compared with 5.82 months (95\% CI: 5.00-6.63) for patients without earlyonset CIN $(P=0.028)$. Multivariate analysis proved timing of CIN was an independent prognostic factor.
Our study showed that early-onset CIN group had a significantly better OS than non-early CIN patients, which is consistent with the conclusion by Jang SH et al, who divided 123 NSCLC patients into early-onset group (within 2 cycles), late-onset group (3-6 cycles) and absence. They found that early-onset neutropenia group showed significantly better PFS and OS than the late-onset group but there was no difference between patients with late-onset and absence of neutropenia group. Intriguingly, it was a new finding that the timing (onset) is a prognostic factor.

As we all known, the majority of patients could experience neutropenia during chemotherapy. Some of them might have better survival than those without CIN. Although CIN certainly is not a direct reason for favorable 
outcome, some studies as well as our findings suggested that CIN might be an indicator for a) the biological activity of cytotoxic drugs, b) patient's genetic predisposition of cytotoxic drugs and c) inflammation level of the patient, which were common factors related to prognosis.

First, the therapeutic effects of patients depend on sufficient biological activity of cytotoxic drugs reaching cancer cell. Although chemotherapy drugs dosing is based on patients' estimated body surface area (BSA)[12, 13], this method has been questioned because of uncertain relationship between BSA and the pharmacokinetics of most cytotoxic agents[14]. Plasma concentration of drugs is affected by patients' different metabolisms, distribution, and catabolism (hepatic and renal blood flow, activity of enzymatic systems). Two decades ago, Gurney $\mathrm{H}$ et al [12] displayed the limitations of BSA dosing which does not account for the whole complex process of cytotoxic drug metabolism. This can lead to an under-dosing of nearly $30 \%$ patients who may have poor oncologic outcome. Moreover, assessment of drug plasma concentration in every patient is too expensive and not practical. Therefore, it might be more convenient to use CIN instead of drug plasma concentration as a predictive factor of efficacy after prospective study validates the results of our study.

Second, the sensitivity of tumor cells to therapeutic drugs is a reflection of the patients' genetic predisposition[15], and theoretically sharing similar features of pharmacokinetics and pharmacodynamics of the regimen in all cells in this patient[14]. Our results showed that early onset CIN indicated a better treatment response, which suggested that patients with early onset neutropenia might be the sensitive population to gemcitabine / gemcitabine-based chemotherapy. On the other hand, multidrug resistance is known as the crossresistance of cancer cells to various cytotoxic drugs which are structurally or functionally unrelated [16]. Either intrinsic resistant or acquired resistant could produce chemotherapeutic failure and malignant tumor progression in cancer [17]. The non-early onset CIN group patients might be resistant to gemcitabine-based chemotherapy intrinsically, and even insensitive to other cytotoxic regimens result in unfavorable outcome. Neutrophil count may be influenced by a host of clinical factors besides performance status, such as age, prior treatments, coexisting infection, and impaired renal or hepatic function [18]. Our multivariate analysis showed strong protective effects of early-onset CIN.

It is not fully understood why such a relationship exists. Although a recent analysis failed to correlate CIN with DNA repair genes, the prognostic significance of CIN may derive from cooperative effects of the repair genes[19]. Thus, patients could respond differently to anti-cancer drugs. The application of neutropenia as a pharmacokinetic marker could be used to individualize the biological effect of patients. Lack of neutropenia may suggest weak biological effect of chemotherapy, which

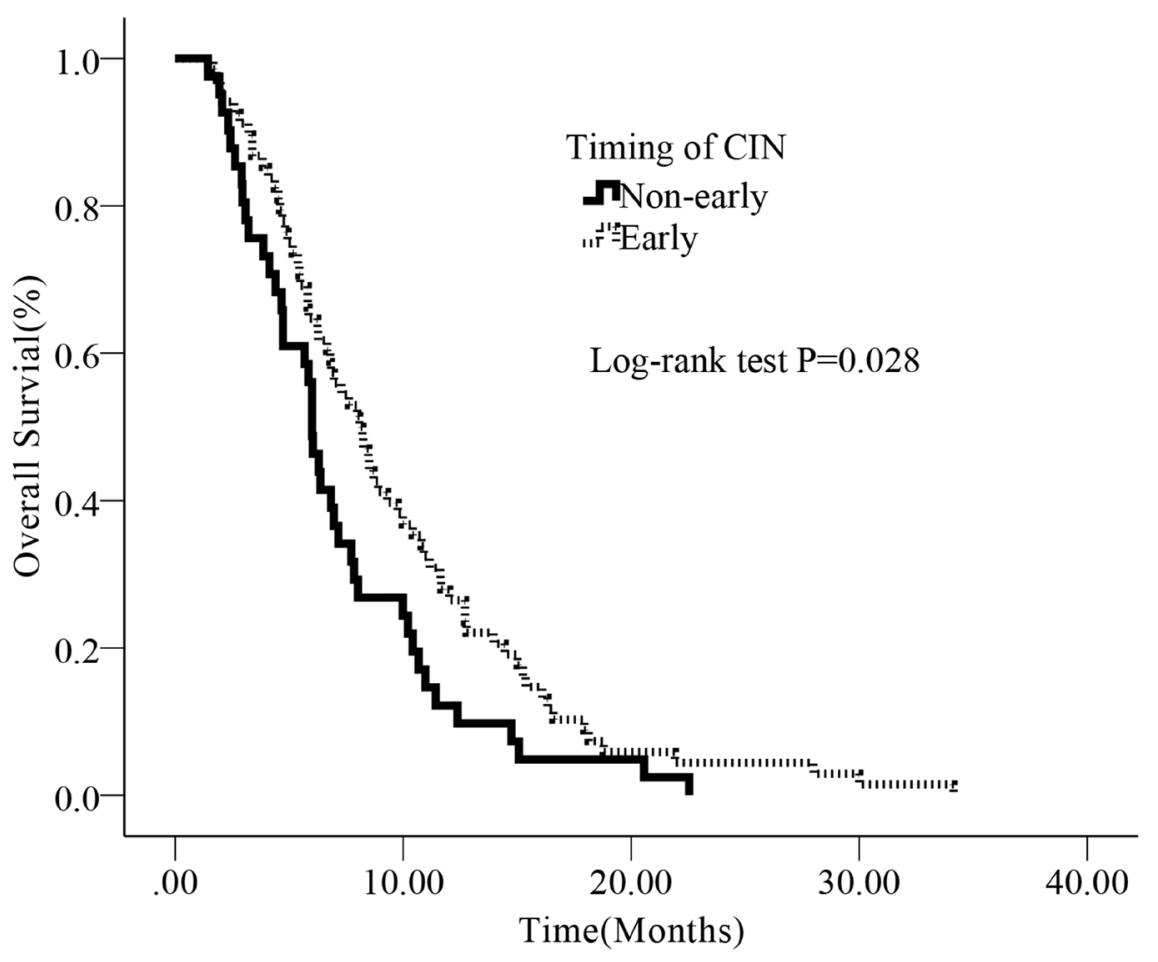

Figure 2: Kaplan-Meier survival curves according to timing of CIN for advanced pancreatic cancer patients. The median OS in the early onset group and non-early onset group were 8.05 months (95\%CI: 5.97-10.13) and 5.82 months (95\% CI: 5.00$6.63)$, respectively. 
Table 2: Univariate analysis for the association between clinical characteristics with survival in advanced pancreatic cancer patients

\begin{tabular}{|c|c|c|c|}
\hline & HR & $95 \% \mathrm{CI}$ & $P$ value \\
\hline \multicolumn{4}{|l|}{ Gender } \\
\hline Male & 1 & & \\
\hline Female & 0.617 & $0.420-0.907$ & 0.018 \\
\hline \multicolumn{4}{|l|}{ Age } \\
\hline$\leq 57$ & 1 & & \\
\hline$>57$ & 1.044 & $0.615-1.774$ & 0.873 \\
\hline \multicolumn{4}{|l|}{ KPS } \\
\hline 90 & 1 & & \\
\hline $70-80$ & 2.376 & $0.868-6.499$ & 0.092 \\
\hline \multicolumn{4}{|l|}{ Pathological differention } \\
\hline Well-moderate & 1 & & \\
\hline Poor & 1.132 & $0.954-1.343$ & 0.043 \\
\hline \multicolumn{4}{|l|}{ Location } \\
\hline Head & 1 & & \\
\hline Body/tail & 1.013 & $0.694-1.480$ & 0.946 \\
\hline \multicolumn{4}{|l|}{ Disease extension } \\
\hline Distant metastasis & 1 & & \\
\hline Locally advanced & 0.693 & $0.447-1.074$ & 0.101 \\
\hline \multicolumn{4}{|c|}{ First-line chemotherapeutic regimens } \\
\hline Gemcitabine monotherapy & 1 & & \\
\hline Gemcitabine and S-1/capecitabine & 0.783 & $0.450-1.363$ & 0.387 \\
\hline Gemcitabine and platinum drugs & 0.522 & $0.219-1.244$ & 0.142 \\
\hline Gemcitabine and nab-paclitaxel & 0.601 & $0.397-0.910$ & 0.016 \\
\hline \multicolumn{4}{|l|}{ Second-line chemotherapy } \\
\hline No & 1 & & \\
\hline Yes & 0.666 & $0.474-0.936$ & 0.019 \\
\hline \multicolumn{4}{|l|}{ Timing of CIN } \\
\hline Non-early & 1 & & \\
\hline Early & 0.644 & $0.441-0.941$ & 0.023 \\
\hline
\end{tabular}

Abbreviation: CIN, chemotherapy-induced neutropenia;

A two-sided significance level of 0.05 was used to evaluate statistical significance.

is possible due to a low dose for an individual patient or patients' intrinsic resistant.

Furthermore, inflammation plays an important role in development and progression of tumor [20]. Changes in hematology ingredients like white blood cells, specifically the neutrophils have been made because of systemic inflammatory response[21]. Neutrophils could promote the acceleration of angiogenesis and suppress the anti-tumor immune response, thereby speed up tumor proliferation [22-24]. In other words, elevated neutrophil counts could promote tumor progression by providing an advantageous environment for proliferation. Jensen HK et al reported that the elevated neutrophils were significantly associated with larger tumor size and worse survival in patients with localized renal cell carcinoma [25]. Another study including 1533 patients with nasopharyngeal cancer (NPC) found that elevated neutropenia predicted poor prognosis [26].Therefore, there is another possible hypothesis for explaining our results that early-onset CIN, decreased neutrophil counts earlier induced by chemotherapy, might change tumor environment by disrupting angiogenesis and releasing immune suppression, and result in favorable clinical outcome.

Based on the three possible mechanisms mentioned above, theoretically occurrence of CIN, especially for early-onset CIN, might be a predictive factor for favorable prognosis $[14,27]$. The significant marker achieved by univariate analysis need to be further validated in the multivariate analysis. Therefore, we think gender is not an independent prognostic factor. In our study, patients with second-line chemotherapy is an independent prognostic factor, there might be the possibility that duration of chemotherapy itself might affect the treatment results. More frequently, most patients could not have the opportunity to receive the second-line chemotherapy due to disease progression, which might be due to the more 
Table 3: Multivariate analysis for the association between clinical characteristics with survival in advanced pancreatic cancer patients

\begin{tabular}{|c|c|c|c|}
\hline & HR & $95 \% \mathrm{CI}$ & P value \\
\hline \multicolumn{4}{|l|}{ Gender } \\
\hline Male & 1 & & \\
\hline Female & 0.680 & $0.443-1.043$ & 0.177 \\
\hline \multicolumn{4}{|l|}{ Age } \\
\hline$\leq 57$ & 1 & & \\
\hline$>57$ & 1.099 & $0.982-1.016$ & 0.914 \\
\hline \multicolumn{4}{|l|}{ KPS } \\
\hline 90 & 1 & & \\
\hline $70-80$ & 1.174 & $1.037-1.419$ & 0.049 \\
\hline \multicolumn{4}{|l|}{ Pathological differention } \\
\hline Well-moderate & 1 & & \\
\hline Poor & 1.159 & $1.078-1.417$ & 0.037 \\
\hline \multicolumn{4}{|l|}{ Disease extension } \\
\hline Distant metastasis & 1 & & \\
\hline Locally advanced & 0.761 & $0.467-1.012$ & 0.087 \\
\hline \multicolumn{4}{|c|}{ First-line chemotherapeutic regimens } \\
\hline Gemcitabine monotherapy & 1 & & \\
\hline Gemcitabine and S-1/capecitabine & 0.966 & $0.548-1.812$ & 0.990 \\
\hline Gemcitabine and platinum drugs & 0.929 & $0.363-2.374$ & 0.878 \\
\hline Gemcitabine and nab-paclitaxel & 0.846 & $0.520-1.376$ & 0.501 \\
\hline \multicolumn{4}{|l|}{ Second-line chemotherapy } \\
\hline No & 1 & & \\
\hline Yes & 0.681 & $0.476-0.972$ & 0.035 \\
\hline \multicolumn{4}{|l|}{ Timing of CIN } \\
\hline Non-early & 1 & & \\
\hline Early & 0.696 & $0.466-0.938$ & 0.027 \\
\hline
\end{tabular}

Hazard ratios of survival and 95\% CI were estimated with Cox's proportional hazards model.

Adjusted for: Gender, Age, KPS, Pathology differention, Disease extension, First-line chemotherapy regimens, Second-line chemotherapy, Timing of CIN

invasive biological behavior of the tumor itself. Therefore, we think it is reasonable that second-line chemotherapy might be an independent prognostic factor.

This study has a few limitations: the retrospective nature, the limited sample size, restriction to Chinese population and confined the chemotherapy to gemcitabine / gemcitabine based chemotherapy. Despite these above, our results have confirmed the use of a potential clinical biomarker (timing of $\mathrm{CIN}$ ) in predicting clinically outcomes. It also has some clinical applications including predicting patients' chemotherapy response, and prognosis, adjusting drug dosage or taking antiinflammatory mediators.

In conclusion, our study suggested that early-onset CIN was a predictive factor for favorable prognosis in patients with advanced pancreatic cancer. We provide a simple, convenient and potential option, using timing of CIN as an indicator, to predict patient's response to chemotherapy early and individualize optimum dosing of drugs. More well-designed and large-scale investigations are warranted to better understand the value of timing of CIN in prognosis of cancer patients.

\section{MATERIALS AND METHODS}

\section{Patients}

The study was comprised of advanced pancreatic cancer patients admitted to Chinese People's Liberation Army (PLA) General Hospital from January $1^{\text {st }} 2008$ to June $1^{\text {st }} 2015$. Our study was approved by the ethics committee of PLA General Hospital. Before the initial time of chemotherapy, written informed and consent were submitted from the patients or their legal guardian. All 
treatments were performed in accordance with relevant guidelines and regulations. Blood samples were collected under institutional review board-approved protocols. Clinical data retrieved electronically from the medical records of PLA General Hospital Registry were reviewed retrospectively.

The inclusion criteria were : 1) patients with cytological or histologic confirmation of pancreatic cancer without any chance of radical operation (including locally advanced and distant metastasis); 2) patients received at least one cycle of gemcitabine / gemcitabine-based chemotherapy as first-line treatment; 3) patients' absolute neutropenia count $(\mathrm{ANC})>2.0 \times 10^{9} / \mathrm{L}$ before treatment; 4) sufficient bone marrow function; 5) normal hepatic and renal function; 6) KPS $\geq 70$; 7) without targeted drugs or other biologics; 8) no bone marrow metastasis; 9) no history of prior chemotherapy for advanced disease or adjuvant therapy within one year; 10) no radiotherapy. Exclusion criteria: 1) no integrated data for toxic effects; 2) no follow-up result. Follow-up evaluations were performed every 3 months. Dates of death were obtained from the China disease prevention and control information system or telephone calls to their families. Medical records were reviewed, and the cause of death was assigned by study physicians. Loss to follow-up refers to patients who became unreachable. We followed up until June 31st, 2016 to obtain clinical and outcome information. Specific details of enrollment and exclusion were also showed in the following flow chart (Figure 1).

\section{Dose intensity of chemotherapy}

Patients in this study all received gemcitabine / gemcitabine-based chemotherapy. Patients had undergone at least one cycle of gemcitabine / gemcitabine (1000 mg/ $\mathrm{m}^{2}, \mathrm{~d} 1,8$, every 3 -week)-based chemotherapy as first-line chemotherapy,

\section{Assessment of neutropenia}

Complete blood cell (CBC) count was checked prior to infusion of agents (Days 1 and 8) and every 7 days. ANC was computed by multiplying the white blood cell count by the total percentage of neutrophils. CIN grading was in accordance with National Cancer Institute (NCI) Common Terminology Criteria for Adverse Events (CTCAE, version 4.0).CIN was also categorized according to time of onset. Early-onset neutropenia was defined as the lowest $\mathrm{ANC}<2.0 \times 10^{9} / \mathrm{L}$ before the end of cycle 2 , nonearly onset CIN defined as the patients had no experience of $\mathrm{ANC}<2.0 \times 10^{9} / \mathrm{L}$ or onset on the end of cycle 2 or after.

\section{Assessment of survival}

OS was defined from date of treatment to death. The primary study endpoint was OS. Censoring occurred if patients were still alive at last follow-up or dead from other diseases.

\section{Statistical analysis}

Data was presented as median (interquartile) for continuous variables, and as frequency or percentage for categorical variables. The Mann-Whitney and chi-square tests were used to determine any statistical difference between the proportions of the two groups. Survival curves of each category were estimated by the KaplanMeier method and compared by the log-rank test. Hazard ratios of survival and 95\% CI were estimated with Cox's proportional hazards model. In COX model we adjusted for: KPS; Pathological differention; disease extension; second-line chemotherapy; timing of CIN.

All of the analyses were performed with the statistical software packages R (http://www.R-project. org, The R Foundation) and EmpowerStats (http://www. empowerstats.com/en/, X\&Y Solutions, Inc., Boston, MA). A two-sided significance level of 0.05 was used to evaluate statistical significance.

\section{CONFLICTS OF INTEREST}

There is no conflict of interest.

\section{REFERENCES}

1. Siegel RL, Miller KD, Jemal A. Cancer statistics, 2016. CA Cancer J Clin. 2016; 66: 7-30. doi: 10.3322/caac.21332.

2. Papadoniou N, Kosmas C, Gennatas K, Polyzos A, Mouratidou D, Skopelitis E, Tzivras M, Sougioultzis S, Papastratis G, Karatzas G, Papalambros E, Tsavaris N. Prognostic factors in patients with locally advanced (unresectable) or metastatic pancreatic adenocarcinoma: a retrospective analysis. Anticancer Res. 2008; 28: 543-9.

3. Han Y, Yu Z, Wen S, Zhang B, Cao X, Wang X. Prognostic value of chemotherapy-induced neutropenia in early-stage breast cancer. Breast Cancer Res Treat. 2012; 131: 483-90. doi: 10.1007/s10549-011-1799-1.

4. Tewari KS, Java JJ, Gatcliffe TA, Bookman MA, Monk BJ. Chemotherapy-induced neutropenia as a biomarker of survival in advanced ovarian carcinoma: an exploratory study of the gynecologic oncology group. Gynecol Oncol. 2014; 133: 439-45. doi: 10.1016/j.ygyno.2014.03.013.

5. Kim YH, Chung HH, Kim JW, Park NH, Song YS, Kang SB. Prognostic significance of neutropenia during adjuvant concurrent chemoradiotherapy in early cervical cancer. J Gynecol Oncol. 2009; 20: 146-50. doi: 10.3802/ 
jgo.2009.20.3.146.

6. Kurihara T, Kogo M, Ishii M, Shimada K, Yoneyama K, Kitamura K, Shimizu S, Yoshida H, Kiuchi Y. Chemotherapy-induced neutropenia as a prognostic factor in patients with unresectable pancreatic cancer. Cancer Chemother Pharmacol. 2015; 76: 1217-24. doi: 10.1007/ s00280-015-2887-4.

7. Liu R, Huang M, Zhao X, Peng W, Sun S, Cao J, Ji D, Wang C, Guo W, Li J, Yin J, Zhu X. Neutropenia predicts better prognosis in patients with metastatic gastric cancer on a combined epirubicin, oxaliplatin and 5-fluorouracil regimen. Oncotarget. 2015; 6: 39018-27. doi: 10.18632/ oncotarget.5730.

8. Di Maio M, Gridelli C, Gallo C, Shepherd F, Piantedosi FV, Cigolari S, Manzione L, Illiano A, Barbera S, Robbiati SF, Frontini L, Piazza E, Ianniello GP, et al. Chemotherapyinduced neutropenia and treatment efficacy in advanced non-small-cell lung cancer: a pooled analysis of three randomised trials. The Lancet Oncology. 2005; 6: 669-77. doi: 10.1016/s1470-2045(05)70255-2.

9. Banerji U, Ashley S, Coward J, Hughes S, Zee Y, Benepal T, Norton A, Eisen T, O'Brien M. The association of chemotherapy induced neutropenia on treatment outcomes in small cell lung cancer. Lung Cancer. 2006; 54: 371-7. doi: 10.1016/j.lungcan.2006.08.001.

10. Yamanaka T, Matsumoto S, Teramukai S, Ishiwata R, Nagai Y, Fukushima M. Predictive value of chemotherapy-induced neutropenia for the efficacy of oral fluoropyrimidine S-1 in advanced gastric carcinoma. Br J Cancer. 2007; 97: 37-42. doi: $10.1038 /$ sj.bjc.6603831.

11. Jang SH, Kim SY, Kim JH, Park S, Hwang YI, Kim DG, Jung KS. Timing of chemotherapy-induced neutropenia is a prognostic factor in patients with metastatic non-small-cell lung cancer: a retrospective analysis in gemcitabine-plusplatinum-treated patients. J Cancer Res Clin Oncol. 2013; 139: 409-17. doi: 10.1007/s00432-012-1341-9.

12. Gurney H. How to calculate the dose of chemotherapy. Br J Cancer. 2002; 86: 1297-302. doi: 10.1038/sj.bjc.6600139.

13. de Jongh FE, Verweij J, Loos WJ, de Wit R, de Jonge MJ, Planting AS, Nooter K, Stoter G, Sparreboom A. Bodysurface area-based dosing does not increase accuracy of predicting cisplatin exposure. J Clin Oncol. 2001; 19: 3733 9.

14. Michael M, Doherty MM. Tumoral drug metabolism: overview and its implications for cancer therapy. J Clin Oncol. 2005; 23: 205-29. doi: 10.1200/jco.2005.02.120.

15. Petros WP, Hopkins PJ, Spruill S, Broadwater G, Vredenburgh JJ, Colvin OM, Peters WP, Jones RB, Hall J, Marks JR. Associations between drug metabolism genotype, chemotherapy pharmacokinetics, and overall survival in patients with breast cancer. J Clin Oncol. 2005; 23: 6117-25. doi: 10.1200/jco.2005.06.075.

16. Chen Z, Shi T, Zhang L, Zhu P, Deng M, Huang C, Hu T, Jiang L, Li J. Mammalian drug efflux transporters of the
ATP binding cassette $(\mathrm{ABC})$ family in multidrug resistance: A review of the past decade. Cancer Lett. 2016; 370: 15364. doi: 10.1016/j.canlet.2015.10.010.

17. Kathawala RJ, Gupta P, Ashby CR Jr, Chen ZS. The modulation of $\mathrm{ABC}$ transporter-mediated multidrug resistance in cancer: a review of the past decade. Drug Resist Updat. 2015; 18: 1-17. doi: 10.1016/j. drup.2014.11.002.

18. Katz OB, Shaked Y. Host effects contributing to cancer therapy resistance. Drug Resist Updat. 2015; 19: 33-42. doi: 10.1016/j.drup.2014.12.002.

19. Szakacs G, Hall MD, Gottesman MM, Boumendjel A, Kachadourian R, Day BJ, Baubichon-Cortay H, Di Pietro A. Targeting the Achilles heel of multidrug-resistant cancer by exploiting the fitness cost of resistance. Chem Rev. 2014; 114: 5753-74. doi: 10.1021/cr4006236.

20. Grivennikov SI, Greten FR, Karin M. Immunity, inflammation, and cancer. Cell. 2010; 140: 883-99. doi: 10.1016/j.cell.2010.01.025.

21. Diakos CI, Charles KA, McMillan DC, Clarke SJ. Cancerrelated inflammation and treatment effectiveness. The Lancet Oncology. 2014; 15: e493-e503. doi: 10.1016/ s1470-2045(14)70263-3.

22. Coussens LM, Werb Z. Inflammation and cancer. Nature. 2002; 420: 860-7. doi: 10.1038/nature01322.

23. Coffelt SB, de Visser KE. Cancer: Inflammation lights the way to metastasis. Nature. 2014; 507: 48-9. doi: 10.1038/ nature 13062.

24. Coussens LM, Zitvogel L, Palucka AK. Neutralizing tumorpromoting chronic inflammation: a magic bullet? Science. 2013; 339: 286-91. doi: 10.1126/science.1232227.

25. Jensen HK, Donskov F, Marcussen N, Nordsmark M, Lundbeck F, von der Maase H. Presence of intratumoral neutrophils is an independent prognostic factor in localized renal cell carcinoma. J Clin Oncol. 2009; 27: 4709-17. doi: 10.1200/jco.2008.18.9498.

26. He JR, Shen GP, Ren ZF, Qin H, Cui C, Zhang Y, Zeng YX, Jia WH. Pretreatment levels of peripheral neutrophils and lymphocytes as independent prognostic factors in patients with nasopharyngeal carcinoma. Head Neck. 2012; 34: 1769-76. doi: 10.1002/hed.22008.

27. Gurney H. Dose calculation of anticancer drugs: a review of the current practice and introduction of an alternative. $\mathrm{J}$ Clin Oncol. 1996; 14: 2590-611. 